\title{
Kribbella karoonensis sp. nov. and Kribbella swartbergensis sp. nov., isolated from soil from the Western Cape, South Africa
}

\author{
Correspondence \\ Paul R. Meyers \\ pmeyers@science.uct.ac.za
}

\author{
Bronwyn M. Kirby, Marilize Le Roes and Paul R. Meyers \\ Department of Molecular and Cell Biology, University of Cape Town, Private Bag 1, \\ Rondebosch, 7701, Cape Town, South Africa
}

\begin{abstract}
Two novel nocardioform actinomycetes, strains $\mathrm{Q} 41^{\top}$ and $\mathrm{HMC}_{2} 5^{\top}$, were isolated from soil samples collected in the Western Cape province, South Africa. Rapid genus identification revealed that the isolates belonged to the genus Kribbella (based on single-digestion restriction analysis of the 16S rRNA gene sequences with Mbol, Vspl, Sphl, SnaBl, Sall and Agel). Both isolates had LL-diaminopimelic acid and glycine in their cell-wall peptidoglycan, and contained mannose and ribose as whole-cell sugars. Strain $\mathrm{HMC}_{2} 5^{\top}$ is able to grow at $45^{\circ} \mathrm{C}$ and in the presence of $\mathrm{NaCl}$ (3\%), cephaloridine $\left(10 \mu \mathrm{g} \mathrm{ml}^{-1}\right)$ and gentamicin sulphate $\left(10 \mu \mathrm{g} \mathrm{ml}^{-1}\right)$. Strain $\mathrm{Q} 41^{\top}$ grows in the presence of $\mathrm{NaCl}(2 \%)$. Neither strain was able to grow under anaerobic conditions, whereas Kribbella flavida KACC $20248^{\top}$, Kribbella jejuensis $\mathrm{HD}^{\top}$, Kribbella koreensis KACC $20250^{\top}$ and Kribbella sandramycini KACC $20249^{\top}$ exhibited weak but distinct growth under anaerobic conditions. Physiological test results and $16 \mathrm{~S}$ rRNA gene sequence analysis allowed $\mathrm{Q} 41^{\top}$ and $\mathrm{HMC} 25^{\top}$ to be distinguished from other members of the genus with validly published names. Strains HMC25 $5^{\top}\left(=\right.$ NRRL B- $24426^{\top}=$ DSM $\left.17345^{\top}\right)$ and ${\text { Q } 41^{\top}}^{\top}\left(=\right.$ NRRL B $-24425^{\top}=$ DSM $17344^{\top}$ ) therefore represent the type strains of novel species, for which the names Kribbella swartbergensis sp. nov. and Kribbella karoonensis sp. nov., respectively, are proposed.
\end{abstract}

The genus Kribbella was established by Park et al. (1999) to accommodate nocardioform actinomycetes that contain LL-diaminopimelic acid in the cell wall. The type species of the genus Hongia, Hongia koreensis, was subsequently transferred to the genus Kribbella (Sohn et al., 2003). With the recent addition of Kribbella lupini (Trujillo et al., 2006), the genus Kribbella currently contains seven species with validly published names (http://www.bacterio.cict.fr/ijk/kribbella. html). Species descriptions of 'Kribbella alba' and 'Kribbella yunnanensis' have been effectively published (Li et al., 2006).

We describe the characterization of two further members of this genus, which were isolated from soil samples collected in Western Cape province, South Africa. The soil from which $\mathrm{Q} 41^{\mathrm{T}}$ was isolated was gathered from the base of a giant quiver tree, Aloe pillansii, growing in the Karoo Desert National Botanical Garden, Worcester. HMC25 $5^{\mathrm{T}}$ was isolated from a soil sample collected from the banks of Gamka River, in Die Hel, situated in the Groot Swartberg mountain range.

The GenBank/EMBL/DDBJ accession numbers for the 16S rRNA gene sequences of strains $\mathrm{O} 41^{\top}$ and $\mathrm{HMC}^{2} 5^{\top}$ are AY995146 and AY995147, respectively.

Scanning electron micrographs of mycelium of strains $\mathrm{Q} 41^{\top}$ and $\mathrm{HMC}^{2} 5^{\top}$ are available as supplementary material in IJSEM Online.
The strains were isolated using two different soil pretreatments. The soil sample from which $\mathrm{HMC} 25^{\mathrm{T}}$ was isolated was initially heated at $120^{\circ} \mathrm{C}$ for $1 \mathrm{~h}$. Approximately $0 \cdot 1 \mathrm{~g}$ soil was then added to $1 \mathrm{ml}$ sterile distilled water and vortexed for $1 \mathrm{~min}$. The sample was then serially diluted in sterile distilled water and spread-plated on MC agar (Nonomura \& Ohara, 1971). The soil sample from which $\mathrm{Q} 41^{\mathrm{T}}$ was isolated was dried at $28^{\circ} \mathrm{C}$ for $24 \mathrm{~h} ; 0 \cdot 1 \mathrm{~g}$ soil was added to $1 \mathrm{ml}$ sterile distilled water and vortexed for $1 \mathrm{~min}$. The soil suspension was then serially diluted in sterile distilled water and spread-plated on PV8 agar (Tormo et al., 2003). All isolation plates contained nalidixic acid (10 $\mu \mathrm{g}$ $\mathrm{ml}^{-1}$ ) and cycloheximide (50 $\mu \mathrm{g} \mathrm{ml}^{-1}$ ) and were incubated at $28{ }^{\circ} \mathrm{C}$ (PV8 plates for 5 days and MC plates for 21 days). Following isolation, both $\mathrm{HMC} 25^{\mathrm{T}}$ and $\mathrm{Q} 41^{\mathrm{T}}$ were maintained on either yeast extract-malt extract agar (ISP 2 medium) (Shirling \& Gottlieb, 1966) or Czapek's solution agar (Atlas, 1993).

Genomic DNA extraction and 16S rRNA gene sequence amplification were performed as previously described by Cook \& Meyers (2003). For rapid genus identification, DNA was amplified using primers F1 and R5, and the resulting amplicons were restricted singly with $\mathrm{MboI}$ (isoschizomer of Sau3AI), VspI (isoschizomer of AsnI), SphI, SnaBI, SalI and AgeI and electrophoresed on a $1.5 \%$ agarose gel. For $16 \mathrm{~S}$ rRNA gene sequence analysis, the PCR product was purified 
using a Cleanmix kit (TA050CLN; Talent) and sequenced. A neighbour-joining phylogenetic tree (Saitou \& Nei, 1987) was constructed using CLUSTAL X, version 1.81 (Thompson et al., 1997), and MEGA, version 2.1 (Kumar et al., 2001).

Selected physiological tests were performed as described by Williams et al. (1989). ISP media were prepared according to the methods of Shirling \& Gottlieb (1966). All physiological tests were incubated at $30^{\circ} \mathrm{C}$ (unless otherwise stated) and the results were recorded after the recommended incubation periods (Shirling \& Gottlieb, 1966; Williams et al., 1989). Kribbella antibiotica YIM $31530^{\mathrm{T}}$ was incubated at room temperature $\left(22^{\circ} \mathrm{C}\right)$. Utilization of carbon sources was tested according to the method of Shirling \& Gottlieb (1966). All carbon sources were tested at $1 \%$ except for sodium acetate, sodium benzoate and sodium citrate, which were tested at $0 \cdot 1 \%$ (all carbon sources were filter-sterilized). Antibiotic susceptibility was tested by incorporating the antibiotics into Bennett's medium (Atlas, 1993) at the given concentrations and incubating at $30{ }^{\circ} \mathrm{C}$ for 14 days. Salt tolerance was tested on ISP 2 (Shirling \& Gottlieb, 1966). Plates were incubated at $30{ }^{\circ} \mathrm{C}$ for 14 days. The ability to grow at $\mathrm{pH}$ 5, 7 and 9 was determined on Bennett's medium plates incubated for 14 days at $30{ }^{\circ} \mathrm{C}$. Degradation of adenine, hypoxanthine and xanthine was determined on modified Bennett's medium plates (Atlas, 1993) incubated at $30^{\circ} \mathrm{C}$ for 21 days. The temperature range for growth was tested (at $20,30,37$ and $45^{\circ} \mathrm{C}$ ) on Bennett's medium plates.

Growth under anaerobic conditions was tested on ATCC medium 172 and ISP 9 with glucose as the sole carbon source (Shirling \& Gottlieb, 1966). Plates were incubated at $25^{\circ} \mathrm{C}$ for 21 days in an anaerobic chamber (model 1024; Forma Scientific) in an atmosphere comprising $\mathrm{H}_{2} / \mathrm{CO}_{2} / \mathrm{N}_{2}$ $(5: 10: 85)$. When plates were first placed in the chamber, each one was opened for $5 \mathrm{~s}$, before being sealed in a plastic bag, to ensure that all residual $\mathrm{O}_{2}$ was removed.

Cultures for chemotaxonomic analysis were grown in ISP 2 in shake flasks for 5 days at $30^{\circ} \mathrm{C}$. Samples were prepared according to the method of Hasegawa et al. (1983), with the exception that freeze-dried cells were used.

Antimicrobial testing against Citrobacter braaki strain 90 (clinical isolate), Enterococcus faecalis (clinical isolate), Escherichia coli ATCC 25922, Klebsiella pneumoniae strain 66 (clinical isolate), Mycobacterium aurum A+, Pseudomonas aeruginosa ATCC 27853 and Staphylococcus aureus ATCC 25923 was performed on 10-day-old stab-inoculated cultures by using standard agar overlays. $\mathrm{HMC}^{\mathrm{T}} 5^{\mathrm{T}}$ and $\mathrm{Q} 41^{\mathrm{T}}$ were stab-inoculated into Czapek's solution agar, Middlebrook 7H9 agar containing $10 \mathrm{mM}$ glucose (Difco; ADC supplement omitted) and ISP 2 agar for antimicrobial testing. For testing against Mycobacterium tuberculosis H37Rv (=ATCC 27294), Kribbella cultures were grown in production medium (Matson \& Bush, 1989) for 5 days at $30^{\circ} \mathrm{C}$. The cultures were filtered through coffee filters (size 102; House of Coffees) and the cells were washed with two culture volumes of deionized water. For each strain, $10 \mathrm{ml}$ methanol was added to the washed cells, which were stirred for $2 \mathrm{~h}$ at room temperature. The methanol extracts were filtered to remove the cells and dried down. Each of these crude extracts was weighed, redissolved in methanol and spotted on silica TLC plates (Merck 1.05554.0001) in a concentration range of 5-60 $\mu \mathrm{g}$ per spot. M. tuberculosis was grown in Middlebrook $7 \mathrm{H} 9$ broth containing the AD supplement (final concentrations: $0.5 \%$ bovine serum albumin, $0 \cdot 2 \%$ glucose) and was incubated as a standing culture at $37^{\circ} \mathrm{C}$ (with intermittent agitation) for 7 days. For antimicrobial testing, the TLC plates were dabbed with the M. tuberculosis culture, using sterile non-absorbent cotton wool, and incubated for $24 \mathrm{~h}$ at $37^{\circ} \mathrm{C}$ in a sealed plastic container containing moist paper towel. Following incubation, the plates were dabbed with $0 \cdot 25 \%$ thiazolyl blue tetrazolium bromide (Sigma) in PBS ( $\mathrm{pH} \mathrm{7 \cdot 3)}$ and incubated for $24 \mathrm{~h}$ at $37^{\circ} \mathrm{C}$ before the results were read. All experiments with $M$. tuberculosis were conducted in a Biosafety Level 3 laboratory.

The restriction fragment patterns indicated that both isolates belonged to the genus Kribbella (see Tables 1, 2 and 3 of Cook \& Meyers, 2003). The lengths of the 16S rRNA gene sequence obtained for $\mathrm{Q} 41^{\mathrm{T}}$ and $\mathrm{HMC}_{2} 5^{\mathrm{T}}$ were 1422 and $1419 \mathrm{bp}$, respectively. A standard nucleotide-nucleotide BLAST search (Altschul et al., 1997) of the GenBank database showed that $\mathrm{HMC}^{\mathrm{T}} 5^{\mathrm{T}}$ was most similar to Kribbella jejuensis strain $\mathrm{HD}^{\mathrm{T}}$ (= KACC $\left.20266^{\mathrm{T}}\right)(97 \%$ similarity over $1430 \mathrm{bp}$ ), while Q41 ${ }^{\mathrm{T}}$ was most similar to Kribbella flavida KACC $20248^{\mathrm{T}}$ and Kribbella sandramycini KACC $20249^{\mathrm{T}}$ (both $98 \%$ over $1421 \mathrm{bp}$ ). The $16 \mathrm{~S}$ rRNA gene sequence similarity between $\mathrm{HMC} 25^{\mathrm{T}}$ and $\mathrm{Q} 41^{\mathrm{T}}$ was $98 \cdot 4 \%$ (determined using DNAMAN, version 4.13; Lynnon Biosoft). These values are low enough to indicate a high probability that the DNA-DNA relatedness between these pairs of strains would be below $70 \%$ (Keswani \& Whitman, 2001; Trujillo et al., 2006). For example, the $16 \mathrm{~S}$ rRNA gene sequence similarity values between Kribbella flavida KACC $20248^{\mathrm{T}}$ and Kribbella solani $\mathrm{DSAl}^{\mathrm{T}}$, between Kribbella jejuensis $\mathrm{HD}^{\mathrm{T}}$ and Kribbella koreensis KACC $20250^{\mathrm{T}}$ and between Kribbella antibiotica YIM $31530^{\mathrm{T}}$ and Kribbella flavida KACC $20248^{\mathrm{T}}$ are $98.6 \%$ (over $1509 \mathrm{nt}$ ), $97 \cdot 8 \%$ (over $1510 \mathrm{nt}$ ) and $98.4 \%$ (over $1427 \mathrm{nt}$ ), respectively (similarities calculated by pairwise global alignment using DNAMAN, version 4.13), yet the reported DNA relatedness values for these pairs of strains are $50 \cdot 4,20 \cdot 9$ and $18 \%$, respectively (Li et al., 2004; Song et al., 2004). As $\mathrm{HMC}^{\mathrm{T}}{ }^{\mathrm{T}}$ and $\mathrm{Q} 41^{\mathrm{T}}$ were phenetically (Table 1) and phylogenetically (Fig. 1) distinct from other kribbellae, DNA-DNA hybridization analysis was not considered necessary to establish these strains as members of novel Kribbella species.

The results of the phenetic characterization of strains $\mathrm{HMC}^{2} 5^{\mathrm{T}}$ and $\mathrm{Q} 41^{\mathrm{T}}$ are presented in Table 1 and in the species descriptions. Although neither $\mathrm{HMC}^{2} 5^{\mathrm{T}}$ nor Q $41^{\mathrm{T}}$ grew anaerobically, it is worth noting that a number of Kribbella species could grow weakly under anaerobic conditions on ATCC medium 172 (Table 1). Interestingly, 


\section{Table 1. Physiological tests that differentiate $\mathrm{HMC}_{2} 5^{\top}$ and $\mathrm{Q} 41^{\top}$ from the type strains of Kribbella species}

Reference strains: 1, Kribbella antibiotica YIM $31530^{\mathrm{T}}$ (unless indicated, data taken from Li et al., 2004); 2, Kribbella flavida KACC 20248 ${ }^{\mathrm{T}}$ (Park et al., 1999); 3, Kribbella jejuensis $\mathrm{HD9}^{\mathrm{T}}$ (Song et al., 2004); 4, Kribbella koreensis KACC 20250 ${ }^{\mathrm{T}}$ (Lee et al., 2000; Sohn et al., 2003); 5, Kribbella lupini LU14 ${ }^{\mathrm{T}}$ (Trujillo et al., 2006); 6, Kribbella sandramycini KACC $20249^{\mathrm{T}}$ (Park et al., 1999); 7, Kribbella solani DSA1 ${ }^{\mathrm{T}}$ (Song et al., 2004). Results that differ from those given in these cited references are given in parentheses. Data for antibiotic susceptibility, growth in the presence of $\mathrm{NaCl}$ and growth on ATCC medium 172 were obtained in this study for all strains unless indicated. Symbols: ++ , good growth; +, growth; +W, weak growth; -, no growth; ND, not determined; NG, no growth on this medium. All strains including the novel strains are positive for growth at $30{ }^{\circ} \mathrm{C}$ and show no growth in the presence of ampicillin, sodium salt $\left(100 \mu \mathrm{g} \mathrm{ml}{ }^{-1}\right)$.

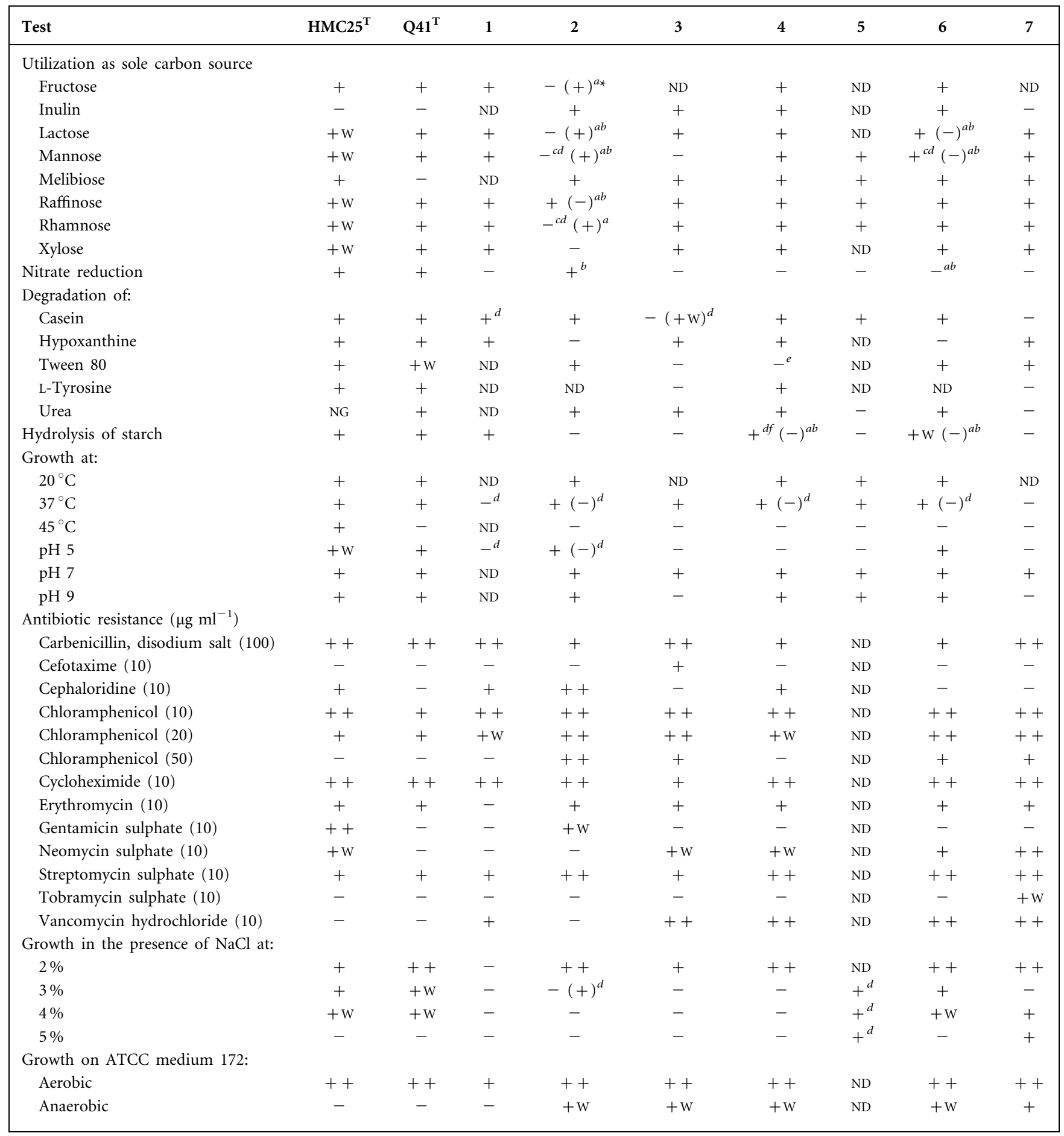

${ }^{\star}$ Data taken from the following sources: a, Li et al. (2004); b, Li et al. (2006); c, Park et al. (1999); d, Trujillo et al. (2006); e, Song et al. (2004); f, Lee et al. (2000) and Sohn et al. (2003). 


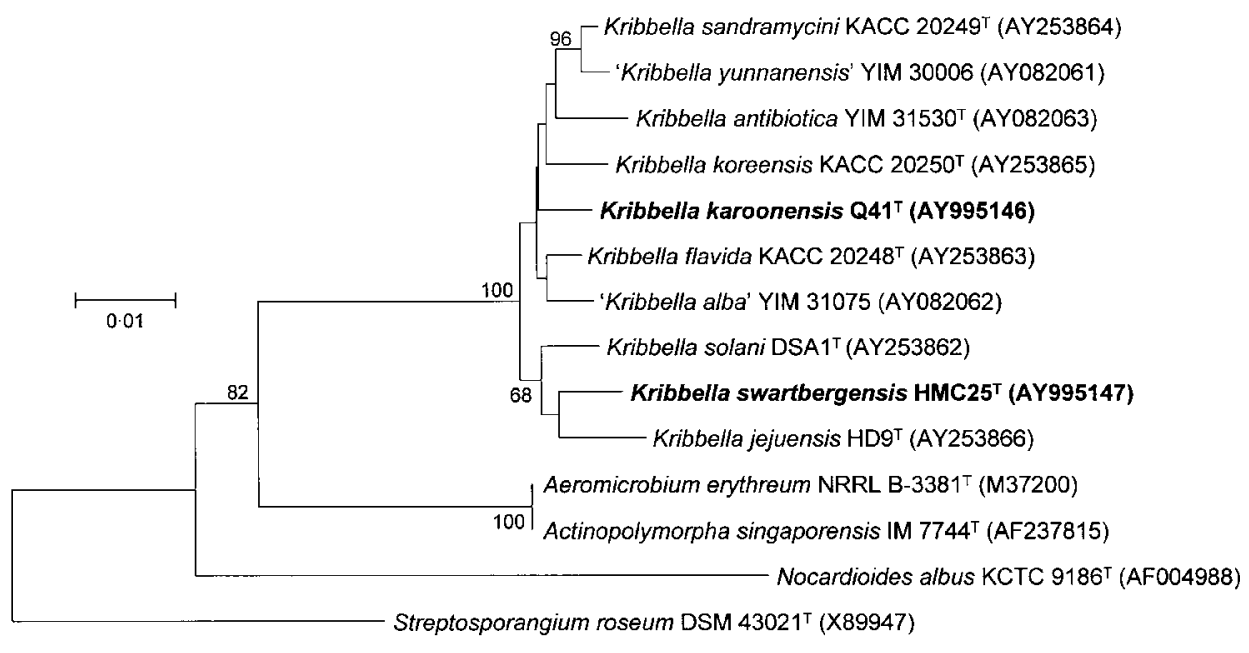

Fig. 1. Unrooted 16S rRNA gene sequence phylogenetic tree showing the relationships between strains $\mathrm{HMC} 25^{\top}$ and $\mathrm{Q} 41^{\top}$ and other related actinomycete taxa. Analysis was conducted over a length of $1412 \mathrm{bp}$ and the tree was constructed using the neighbour-joining method, with bootstrap percentages based on 1000 resampled datasets. Streptosporangium roseum DSM $43021^{\top}$ was set as the outgroup.

Kribbella solani $\mathrm{DSA}^{\mathrm{T}}$ grew moderately well under anaerobic conditions, but not as well as on the control plate incubated under aerobic conditions. None of the kribbellae grew on ISP 9 under anaerobic conditions.

Under a scanning electron microscope, the aerial mycelium of $\mathrm{Q} 41^{\mathrm{T}}$ appeared as a fine mesh of intertwined mycelia, which formed buds at the tips (see left-hand image of Supplementary Fig. S1 in IJSEM Online). The mycelia on the agar surface fragmented into rod-shaped elements (Supplementary Fig. S1; right-hand image). $\mathrm{HMC}^{\mathrm{T}} \mathrm{T}^{\mathrm{T}}$ also appeared as fine aerial mycelium, but budding was not present (Supplementary Fig. S2). The aerial mycelium of both isolates fragmented into rod-shaped elements.

Neither $\mathrm{HMC}^{\mathrm{T}} 5^{\mathrm{T}}$ nor $\mathrm{Q} 41^{\mathrm{T}}$ showed antibiotic activity against any of the test bacteria.

\section{Description of Kribbella swartbergensis sp. nov.}

Kribbella swartbergensis (swart'berg.en'sis. N.L. fem. adj. swartbergensis pertaining to the Groot Swartberg mountain range, South Africa).

Gram-positive. Aerobic. Aerial mycelium is white and the vegetative hyphae are cream-coloured on inorganic salts/ starch agar (ISP 4). Hyphae fragment into rod-shaped elements in broth and on agar. No diffusible pigments are produced on glycerol-asparagine agar (ISP 5). Melanin pigment is not produced on peptone-yeast extract-iron agar (ISP 6) or tyrosine agar (ISP 7). Nitrate is reduced. Catalase-positive. Produces $\mathrm{H}_{2} \mathrm{~S}$. Utilizes $\mathrm{D}(-)$-fructose, $\mathrm{D}(+)$-glucose, maltose, $\mathrm{D}(+)$-melibiose, $\mathrm{D}(-)$-sorbitol and sucrose as sole carbon sources, with weak growth on L ( + )-arabinose, glycerol, myo-inositol, lactose, D-mannitol,
$\mathrm{D}(+)$-mannose, raffinose, $\mathrm{L}(+)$-rhamnose, sodium acetate, sodium citrate, sodium lactate, sodium succinate and $\mathrm{D}(+)$-xylose after 21 days. Grows at $20-45^{\circ} \mathrm{C}$. Grows at pH 7 and 9. Aesculin, arbutin and starch are hydrolysed. Degrades adenine, casein, gelatin, hypoxanthine, Tween 80 and L-tyrosine. Grows well on ISP 2 medium in the presence of $3 \% \mathrm{NaCl}$, with weak growth at $4 \% \mathrm{NaCl}$. Grows in the presence of carbenicillin disodium salt $\left(100 \mu \mathrm{g} \mathrm{ml}^{-1}\right)$, cephaloridine $\left(10 \mu \mathrm{g} \mathrm{ml}^{-1}\right)$, chloramphenicol $\left(20 \mu \mathrm{g} \mathrm{ml}^{-1}\right)$, cycloheximide $\left(10 \mu \mathrm{g} \mathrm{ml}^{-1}\right)$, erythromycin $\left(10 \mu \mathrm{g} \mathrm{ml}^{-1}\right)$, gentamicin sulphate $\left(10 \mu \mathrm{g} \mathrm{ml}^{-1}\right)$, neomycin sulphate $\left(10 \mu \mathrm{g} \mathrm{ml}^{-1}\right)$ and streptomycin sulphate $\left(10 \mu \mathrm{g} \mathrm{ml}^{-1}\right)$ The cell-wall peptidoglycan contains LL-diaminopimelic acid and glycine (chemotype I). The whole-cell sugars are ribose and mannose.

The type strain, $\mathrm{HMC}^{\mathrm{T}} 5^{\mathrm{T}}\left(=\mathrm{NRRL} \quad \mathrm{B}-24426^{\mathrm{T}}=\mathrm{DSM}\right.$ $17345^{\mathrm{T}}$ ), was isolated from soil from the banks of the River Gamka, Die Hel, Groot Swartberg mountain range, Western Cape province, South Africa.

\section{Description of Kribbella karoonensis sp. nov.}

Kribbella karoonensis (ka.roo.nen'sis. N.L. fem. adj. karoonensis pertaining to the Karoo Desert National Botanical Garden in Worcester, South Africa).

Gram-positive. Aerobic. Aerial mycelium is pale cream/ white and the vegetative hyphae are cream/yellow on inorganic salts/starch agar (ISP 4). Hyphae fragment into rod-shaped elements in broth and on agar. No diffusible pigments are produced on glycerol/asparagine agar (ISP 5). Melanin pigment is not produced on peptone/yeast extract/ iron agar (ISP 6) or tyrosine agar (ISP 7). Nitrate is reduced. Catalase-positive. Produces $\mathrm{H}_{2} \mathrm{~S}$. Utilizes $\mathrm{D}(-)$-fructose, 
$\mathrm{D}(+)$-glucose, glycerol, myo-inositol, lactose, D-mannitol, $\mathrm{D}(+)$-mannose, raffinose, $\mathrm{L}(+)$-rhamnose, sodium succinate, sucrose and $\mathrm{D}(+)$-xylose as sole carbon sources, with doubtful growth on adonitol, $\mathrm{L}(+)$-arabinose, maltose, sodium acetate, sodium benzoate, sodium citrate and sodium lactate. Grows at $20-37^{\circ} \mathrm{C}$. Grows at $\mathrm{pH} 5,7$ and 9. Aesculin, arbutin and starch are hydrolysed. Degrades adenine, casein, gelatin, hypoxanthine and L-tyrosine. Produces weak degradation of Tween 80 after 14 days, but the type strain grows poorly on this test medium. Grows well on ISP 2 medium in the presence of $2 \% \mathrm{NaCl}$, with weak growth in the presence of 3 and $4 \% \mathrm{NaCl}$. Grows in the presence of carbenicillin disodium salt $\left(100 \mu \mathrm{g} \mathrm{ml}^{-1}\right)$, chloramphenicol $\left(20 \mu \mathrm{g} \mathrm{ml}^{-1}\right)$, cycloheximide $\left(10 \mu \mathrm{g} \mathrm{ml}^{-1}\right)$, erythromycin $\left(10 \mu \mathrm{g} \mathrm{ml}^{-1}\right)$ and streptomycin sulphate $\left(10 \mu \mathrm{g} \mathrm{ml}^{-1}\right)$. Cell-wall peptidoglycan contains LL-diaminopimelic acid and glycine (chemotype I). The whole-cell sugars are ribose and mannose.

The type strain Q41 ${ }^{\mathrm{T}}\left(=\mathrm{NRRL} B-24425^{\mathrm{T}}=\mathrm{DSM} 17344^{\mathrm{T}}\right)$, was isolated from soil collected from the base of a giant quiver tree, Aloe pillansii, growing in the Karoo Desert National Botanical Garden, Worcester, Western Cape province, South Africa.

\section{Acknowledgements}

The authors wish to thank Di James for DNA sequencing, Miranda Waldron of the Electron Microscope Unit, University of Cape Town (UCT), for assistance with scanning electron microscopy, Vanessa Davids for providing the clinical bacteria for antibiotic testing and Professor Dr Hans G. Trüper for his assistance in deriving the specific names for the isolates. We thank Professor Lafras Steyn of the Institute of Infectious Diseases and Molecular Medicine at University of Cape Town for the use of the BL3 Laboratory and Associate Professors Shez Reid and Val Abratt for use of the anaerobic chamber. B. M. K. holds a local post-graduate scholarship from the Medical Research Council of South Africa (MRC). M.L.R. holds a Scarce Skills Scholarship from the National Research Foundation (NRF) and a UCT Postgraduate Research Associateship. This work was supported by research grants to P.R.M. from the MRC, the NRF (grant no. 2054264) and the University Research Committee (UCT).

\section{References}

Altschul, S. F., Madden, T. L., Schäffer, A. A., Zhang, J., Zhang, Z., Miller, W. \& Lipman, D. J. (1997). Gapped BLAST and PSI-BLAST: a new generation of protein database search programs. Nucleic Acids Res 25, 3389-3402.

Atlas, R. M. (1993). Handbook of Microbiological Media. Edited by L. C. Parks. Boca Raton, FL: CRC Press.

Cook, A. E. \& Meyers, P. R. (2003). Rapid identification of filamentous actinomycetes to the genus level using genus-specific $16 \mathrm{~S}$ rRNA gene restriction fragment patterns. Int J Syst Evol Microbiol 53, 1907-1915.
Hasegawa, T., Takizawa, M. \& Tanida, S. (1983). A rapid analysis for chemical grouping of aerobic actinomycetes. J Gen Appl Microbiol 29, 319-322.

Keswani, J. \& Whitman, W. B. (2001). Relationship of $16 \mathrm{~S}$ rRNA sequence similarity to DNA hybridization in prokaryotes. Int J Syst Evol Microbiol 51, 667-678.

Kumar, S., Tamura, K., Jakobsen, I. B. \& Nei, M. (2001). MEGA2: molecular evolutionary genetics analysis software. Arizona State University, Tempe, USA. http://www.megasoftware.net

Lee, S. D., Kang, S.-O. \& Hah, Y. C. (2000). Hongia gen. nov., a new genus of the order Actinomycetales. Int J Syst Evol Microbiol 50, 191-199.

Li, W. J., Wang, D., Zhang, Y. Q., Schumann, P., Stackebrandt, E., Xu, L. H. \& Jiang, C. L. (2004). Kribbella antibiotica sp. nov., a novel nocardioform actinomycete strain isolated from soil in Yunnan, China. Syst Appl Microbiol 27, 160-165.

Li, W. J., Wang, D., Zhang, Y. Q., Xu, L. H. \& Jiang, C. L. (2006). Kribbella yunnanensis sp. nov., Kribbella alba sp. nov., two novel species of genus Kribbella isolated from soils in Yunnan, China. Syst Appl Microbiol 29, 29-35.

Matson, J. A. \& Bush, J. A. (1989). Sandramycin, a novel antitumor antibiotic produced by a Nocardioides sp. J Antibiot 42, 1763-1767.

Nonomura, H. \& Ohara, Y. (1971). Distribution of actinomycetes in soil. VIII. Green spore group of Microtetraspora, its preferential isolation and taxonomic characteristics. J Ferment Technol 49, 1-7.

Park, Y. H., Yoon, J. H., Shin, Y. K., Suzuki, K., Kudo, T., Seino, A., Kim, H. J., Lee, J. S. \& Lee, S. T. (1999). Classification of 'Nocardioides fulvus' IFO 14399 and Nocardioides sp. ATCC 39419 in Kribbella gen. nov., as Kribbella flavida sp. nov. and Kribbella sandramycini sp. nov. Int J Syst Bacteriol 49, 743-752.

Saitou, N. \& Nei, M. (1987). The neighbor-joining method: a new method for reconstructing phylogenetic trees. Mol Biol Evol 4, 406-425.

Shirling, E. B. \& Gottlieb, D. (1966). Methods for characterization of Streptomyces species. Int J Syst Bacteriol 16, 313-340.

Sohn, K., Hong, S. G., Bae, K. S. \& Chun, J. (2003). Transfer of Hongia koreensis Lee et al. 2000 to the genus Kribbella Park et al. 1999 as Kribbella koreensis comb. nov. Int J Syst Evol Microbiol 53, 1005-1007.

Song, J., Kim, B. Y., Hong, S. B., Cho, H. S., Sohn, K., Chun, J. \& Suh, J. W. (2004). Kribbella solani sp. nov. and Kribbella jejuensis sp. nov., isolated from potato tuber and soil in Jeju, Korea. Int J Syst Evol Microbiol 54, 1345-1348.

Thompson, J. D., Gibson, T. J., Plewniak, F., Jeanmougin, F. \& Higgins, D. G. (1997). The CLUSTAL_X windows interface: flexible strategies for multiple sequence alignment aided by quality analysis tools. Nucleic Acids Res 25, 4876-4882.

Tormo, J. R., García, J. B., DeAntonio, M., Feliz, J., Mira, A., Díez, M. T., Hernández, P. \& Peláez, F. (2003). A method for the selection of production media for actinomycete strains based on their metabolite HPLC profiles. J Ind Microbiol Biotechnol 30, 582-588.

Trujillo, M. E., Kroppenstedt, R. M., Schumann, P. \& MartínezMolina, E. (2006). Kribbella lupini sp. nov., isolated from the roots of Lupinus angustifolius. Int J Syst Evol Microbiol 56, 407-411.

Williams, S. T., Goodfellow, M. \& Alderson, G. (1989). Genus Streptomyces Waksman and Henrici 1943, 339 AL. In Bergey's Manual of Systematic Bacteriology, vol. 4, pp. 2452-2492. Edited by S. T. Williams, M. E. Sharpe \& J. G. Holt. Baltimore: Williams \& Wilkins. 